\title{
Interfaces da flexibilidade cognitiva e da aprendizagem em fóruns de discussão
}

\section{Interfaces of cognitive flexibility and learning in discussion forums}

\author{
Eduardo Rodrigues da Silva \\ Silvia Dotta \\ Universidade Federal do ABC, UFABC (Brasil)
}

\section{Resumo}

Este artigo tem como objetivo apresentar a revisão sistemática da literatura sobre a Flexibilidade Cognitiva enquanto capacidade de inibir uma resposta, mudar de opinião ou atitude por outra mais adequada. Esta característica do comportamento humano está presente na teoria da aprendizagem desenvolvida por Spiro e seus colaboradores, mas também nas funções executivas da neuropsicologia cognitiva. A elaboração de atividades para fóruns de discussão baseadas nestes princípios pode fornecer a base conceitual para que os professores possam estimular o enfrentamento de casos ou situações-problema, de modo a promover a flexibilização do pensamento, a aprendizagem ativa e o protagonismo dos estudantes, baseado em um conjunto de conhecimentos já aprendidos anteriormente. Utilizamos como fonte de pesquisa 12 bases de dados, resultando em um total de 224 documentos encontrados, dentre os quais 56 foram selecionados como pertinentes para o presente estudo, após a aplicação dos critérios de exclusão. A Flexibilidade Cognitiva foi apontada como uma teoria de aprendizagem capaz de desenvolver a habilidade de flexibilizar o pensamento e os construtos cognitivos que promovem o conhecimento dos estudantes, ajudando-os a compreender melhor as convenções sociais e interferências tecnológicas que conjugam textos verbais e não verbais às mais variadas expressões matemáticas, gráficas, diagramáticas e esquemáticas, bem como a apreensão dos caminhos resolutivos de um caso ou situação-problema.

Palavras-chave: flexibilidade; cognição; fórum de discussões; atividades de aprendizagem; resolução de problemas.

\begin{abstract}
This work aims to present a systematic review of the literature on Cognitive Flexibility as the ability to inhibit a response, change an opinion or attitude by another more appropriate. This feature of human behavior is present in the learning theory developed by Spiro and colleagues, as well as in the executive functions cognitive neuropsychology. The activities prepared for discussion forums based on these principles can provide the conceptual basis for teachers to stimulate coping cases or problem situations, in order to promote the flexibility of thinking, active learning and the role of students, based on a body of knowledge already seized earlier. We used as a source of search 12 databases, resulting in a total of 224 documents
\end{abstract}


founded, of which 56 were selected as relevant to the present study, after application of the exclusion criteria. Cognitive Flexibility was appointed as a learning theory able to develop the ability of flexible thinking and cognitive constructs that promote the knowledge of the students, helping them to better understand the social conventions and technological interference that combine verbal and non-verbal texts with various mathematical, graphic, diagrammatic and schematic expressions, as well as the apprehension of resolute way of a case or problem situation.

Keywords: flexibility; cognition; discussion forum; learning activities; problem solving.

Segundo Spiro et al. (1992), flexibilidade cognitiva significa a capacidade de os sujeitos adaptarem o conhecimento adquirido para conseguir fornecer uma resposta alternativa a uma situação não vivenciada anteriormente.

Nesta pesquisa, nosso objetivo foi responder à seguinte questão: Atividades de ensino e aprendizagem baseadas em flexibilidade cognitiva podem facilitar a construção do conhecimento em fóruns de discussão? Verificamos que os fóruns de discussão são ferramentas de grande potencial interativo para promover a troca de conhecimentos e pontos de vista entre estudantes e professores nos Ambientes Virtuais de Ensino e Aprendizagem (AVEA). No entanto, encontramos poucos documentos dedicados a estudar a elaboração de atividades para fóruns de discussão baseados em Flexibilidade Cognitiva e no enfrentamento de problemas, reais ou potenciais, por parte de estudantes e professores.

Nesta revisão da literatura priorizamos o estudo do referencial teórico, bem como os objetivos e resultados, dos documentos encontrados. O percurso metodológico adotado foi o da revisão sistemática integrativa, que consistiu na elaboração da questão de pesquisa; elaboração das palavras-chave e expressão de busca; recuperação de documentos em doze bases de dados diferentes; aplicação de critérios de exclusão; leitura integral e definição dos documentos pertinentes e não pertinentes.

\section{A Flexibilidade Cognitiva e sua relação com as atividades de ensino e aprendizagem}

Para Spiro et al. (1987), a mobilização de conhecimentos prévios para resolução de problemas e tomada de decisões é um princípio fundamental de todas as teorias cognitivas. No entanto, explica que pouco se sabe sobre uma metodologia de organização e aplicação do conhecimento que vise facilitar o enfrentamento de novas situações, especialmente em condições pouco estruturadas ou com informações de naturezas diferentes e não lineares, nas quais se exija uma flexibilidade cognitiva capaz de adaptar, reestruturar ou transferir fontes diferentes de informação na memória do estudante (domínios do conhecimento). 
Conforme observam Spiro et al. (1992) e Carvalho (2000; 2002; 2008), a Teoria da Flexibilidade Cognitiva (TFC) não pode ser considerada uma teoria geral, sendo indicada para aplicação no nível avançado de aquisição do conhecimento. Há três níveis sequenciais: 1) introdutório, ou de iniciação, no qual os estudantes adquirem conhecimentos acerca do modo de operacionalização de fórmulas e conceitos humanos básicos; 2) avançado, no qual os estudantes usam os conhecimentos adquiridos no nível introdutório para resolver casos ou situações-problema reais ou semelhantes ao cotidiano: menos estruturados, não uniformes, imprecisos, aparentemente não coesos e com pouco relacionamento entre os domínios do conhecimento; 3) especialização, que exige experiência e prática no campo de atuação.

Para Spiro et al. (1987; 1992), uma falha comum no desenho instrucional das atividades de educação em níveis mais avançados é o modo exageradamente simplificado e bem estruturado de representação dos domínios do conhecimento, com o intuito de facilitar o ensino dos conteúdos de aprendizagem, produzindo um aprendizado de natureza irrealista e contextualmente desconectado da realidade. Na TFC é importante que o enunciado dos casos ou situações-problema possua informações suficientes para os estudantes compreenderem a natureza do que deva ser resolvido em conjunto, preservando lacunas e múltiplas representações dos conceitos, de modo que percebam ativamente as diferentes formas combinatórias de representação e aplicação em contextos reais. A apresentação de exemplos deve considerar perspectivas, dimensões, conexões, irregularidades e domínios do conhecimento diferentes e variados, visando estimular a transferência e o interrelacionamento de informações, bem como a estruturação de esquemas resolutivos.

\section{A Flexibilidade Cognitiva e a sua relação com a neuropsicologia cognitiva}

A relação entre TFC e a neuropsicologia (ou neurociência) cognitiva não foi o objetivo de nossa pesquisa. No entanto, a relevância de resultados sobre a capacidade humana de flexibilizar o pensamento, transferir e adaptar conhecimentos prévios para a resolução de novas situações exigiu a nossa atenção.

A capacidade humana de pensamento abstrato e flexibilidade cognitiva faz parte de um construto de propriedades comportamentais nobres chamadas de Funções Executivas (FE), descritas como processos cognitivos superiores extremamente refinados e diretamente relacionados ao funcionamento do Córtex Pré-frontal (CPF), região do cérebro que executa importante papel na administração e regulação do comportamento humano (Debiase, 2013; Zuanetti, 2015). As faculdades mentais são operacionalizadas em diferentes regiões do cérebro, conectadas funcionalmente entre si, como uma espécie de "orquestra hierárquica" (Paleari, 2013), como exemplificado na figura 1. 
Figura 1. Representação dos lobos cerebrais

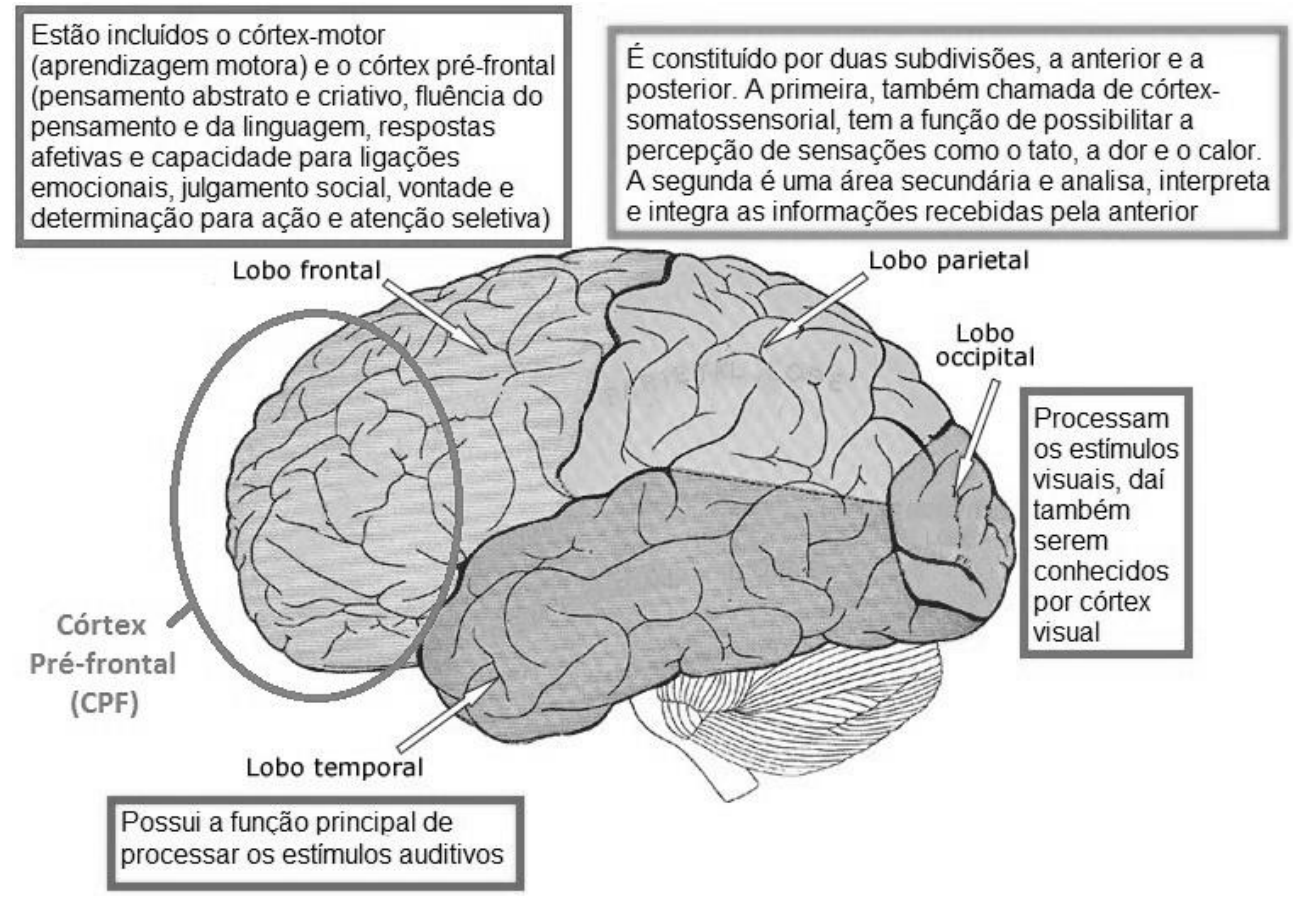

Fonte: Oliveira, 2013.

As FE se referem a um conjunto de habilidades comportamentais críticas que controlam os nossos pensamentos, emoções e ações e são divididas em três categorias: 1) autocontrole: capacidade de resistir a uma tentação, agir menos impulsivamente e manter a concentração em uma tarefa; 2) memória de trabalho: capacidade de manter informações na mente para serem acessadas e manipuladas e 3) flexibilidade cognitiva: capacidade de usar o pensamento criativo e realizar ajustes flexíveis para se adaptar às mudanças e resolver problemas (Morton, 2013; Diamond, 2007).

Segundo Junior e Melo (2011), o estudo da relação entre a cognição e o comportamento neuropsicológico humano é relativamente novo para a ciência. Ainda assim, verificamos que ambas estudam a memória, o pensamento e os mecanismos que atuam na resolução de uma tarefa ou tomada de decisão, permitindo ao indivíduo flexibilizar o conhecimento prévio e assimilar uma nova informação. Ambas também pesquisam a interação entre a experiência individual e os relacionamentos sociais na aprendizagem, o que sugere a validade de se aprofundar os estudos comparativos entre as teorias da aprendizagem e a neuropsicologia cognitiva. 


\section{Pontos possíveis de convergência no estudo da Flexibilidade Cognitiva}

A Teoria da Aprendizagem Significativa de David Ausubel (TAS); a Teoria da Zona de Desenvolvimento Proximal de Vigotski (ZDP); e a TFC convergem em um mesmo aspecto central: o conhecimento prévio dos estudantes deve ser a matériaprima no desenvolvimento de novos conhecimentos, mediados por uma pessoa mais experiente e construído a partir da inter-relação social, em oposição à absorção de conhecimentos prontos ou a sua memorização.

A TAS propõe que um conhecimento novo, para ser assimilado significativamente pelo estudante, deve ancorar-se nos conhecimentos prévios, chamados de subsunçores. Para Ausubel (2003), a aquisição significativa de conhecimentos também deve promover o desenvolvimento de um mecanismo de aprendizagem que considere a estrutura cognitiva do estudante, isto é, a sua disposição ou capacidade para relacionar os conhecimentos novos com os já assimilados. Observa, ainda, a necessidade dos conteúdos e ações pedagógicas seguirem um planejamento nãoarbitrário (ou seja: intencional, sensível, não-aleatório) e não-literal (ou seja: substantivo, lógico). Acreditamos que a TFC possa colaborar com a TAS ao estimular a generalização do conhecimento subsunçor para ancorar o novo conceito, bem como desenvolver a capacidade de integração flexível entre ambos, respeitando a estrutura cognitiva prévia de cada estudante.

De acordo com Vigotski (1996), a ZDP é a base do desenvolvimento da aprendizagem e corresponde à distância entre o nível de desenvolvimento real (capacidade de o sujeito resolver problemas de modo autônomo) e o nível de desenvolvimento próximo (capacidade de o sujeito solucionar problemas auxiliado por alguém mais experiente), dando origem às funções psicológicas superiores. Vigotski (2008) aponta, também, que o desenvolvimento do pensamento é determinado pela linguagem e pela experiência sociocultural do estudante, mediante compreensão e assimilação da realidade complexa. Ao relacionar a ZDP com a TFC, compreendemos a importância dos professores mediarem a resolução contextualizada e sócio-construída de casos ou situações-problema, promovendo o desenvolvimento dos mecanismos cognitivos flexíveis de inter-relacionamento entre aquilo que o estudante sabe e aquilo que precisa saber. Quando o conhecimento já está estruturado, a adaptação cognitiva a novas situações pode ser uma tarefa difícil, exigindo dos estudantes uma grande capacidade de flexibilizar a estrutura mental e transferir domínios do conhecimento.

\section{A Flexibilidade Cognitiva e sua aplicabilidade na elaboração de atividades para fóruns de discussão}

Acreditamos que a TFC seja útil para trabalhar conceitos com variáveis múltiplas de informação e que exijam respostas adaptadas ao contexto ou situação no âmbito dos 
fóruns de discussão. Para Lima (2002), o fórum de discussão constitui uma das mais importantes ferramentas de interação e construção colaborativa do conhecimento nos AVEA, permitindo compreender a importância do que se sabe como ponto de partida para a mediação e construção coletiva de novos conhecimentos, por meio da troca de informações entre os sujeitos da educação (tutores, estudantes e equipe didático-pedagógica). Estas características estão ilustradas na Figura 2, tendo por base um modelo baseado na ZDP de Vigotski.

Nesta pesquisa do "estado da arte" não encontramos exemplos relevantes de aplicação da flexibilidade cognitiva em fóruns de discussão. Assim, formulamos uma proposta de estudo empírico da TFC para aplicação em um fórum de discussão do curso Antártica ou Antártida? Como inserir as ciências polares no currículo escolar, voltado para um grupo de estudantes que também são professores da rede municipal de São Bernardo do Campo e Santo André, cidades da região do ABC Paulista. O curso foi estruturado no AVEA Moodle da Universidade Federal do ABC e teve duração total de dez semanas, iniciando em 26 de setembro de 2016. O fórum de discussão em análise durou três semanas.

Figura 2. Representação da interação no Fórum de Discussão e sua relação com a ZDP de Vigotski

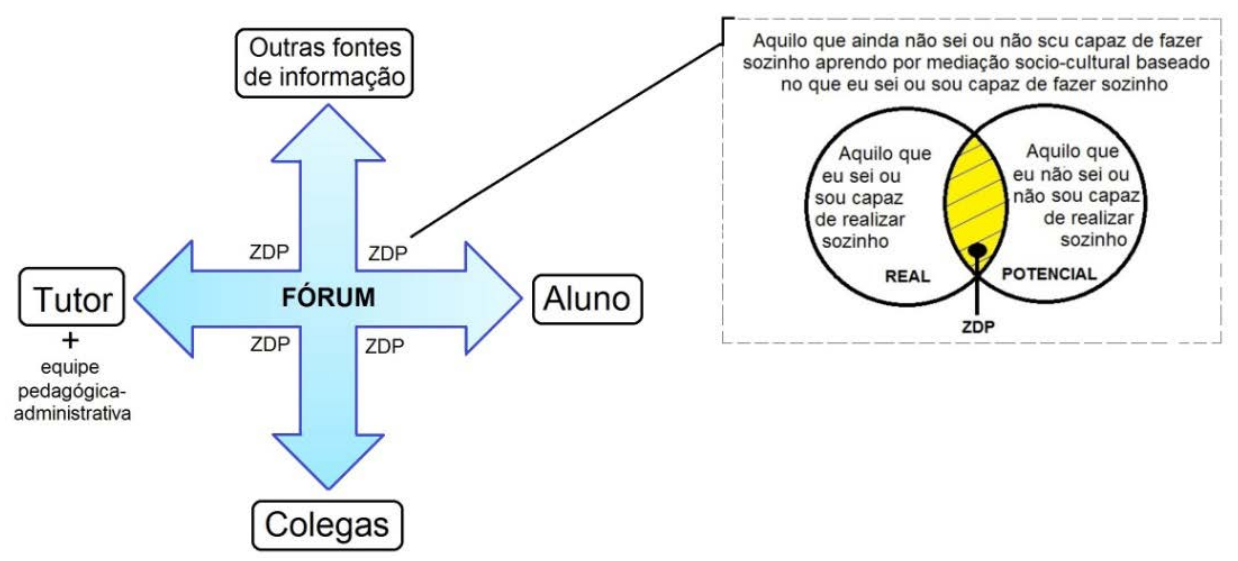

Adaptado de: Lima, 2002, p. 89.

Para compreendermos melhor os resultados, dividimos os estudantes em dois tópicos de discussão (grupos A e B) e aplicamos a metodologia proposta em apenas um deles. $\mathrm{O}$ mesmo caso foi problematizado em ambos, visando assegurar a neutralidade do estudo. Para estimular o debate de ideias, tornamos a dividir os estudantes em dois subgrupos: um responsável por defender a tese da Amazônia como o pulmão do mundo e o outro, a Antártica. O conhecimento a ser desenvolvido 
foi reformulado à luz da TFC: propor um problema para discussão (quem é o pulmão do mundo: a Amazônia ou a Antártica?); realizar múltiplas representações do conhecimento, com bases de natureza diferentes (poesias, infográficos, ilustrações, notícias); apresentar a informação de modo não linear e com lacunas propositais, para estimular a interação, a pesquisa e a discussão; mediar a construção do conhecimento.

Realizamos uma pesquisa voluntária e anônima com os estudantes para observar os resultados. O grupo em que a metodologia proposta foi empregada (Grupo A) apresentou um número de postagens no fórum superior ao grupo em que ela foi suprimida (Grupo B). Os estudantes do Grupo A também reconheceram mais positivamente aspectos como relevância das contribuições, qualidade na interação, acolhimento, compartilhamento de saberes e a importância dos recursos audiovisuais na representação do conhecimento. Os resultados estão representados na figura 3. Enfatizamos que os dados apresentados neste estudo empírico são preliminares e fazem parte de um projeto maior de pesquisa que não foram o objetivo deste levantamento da literatura.

Figura 3. Gráfico de pesquisa de opinião com os estudantes sobre a aquisição de conhecimentos no fórum

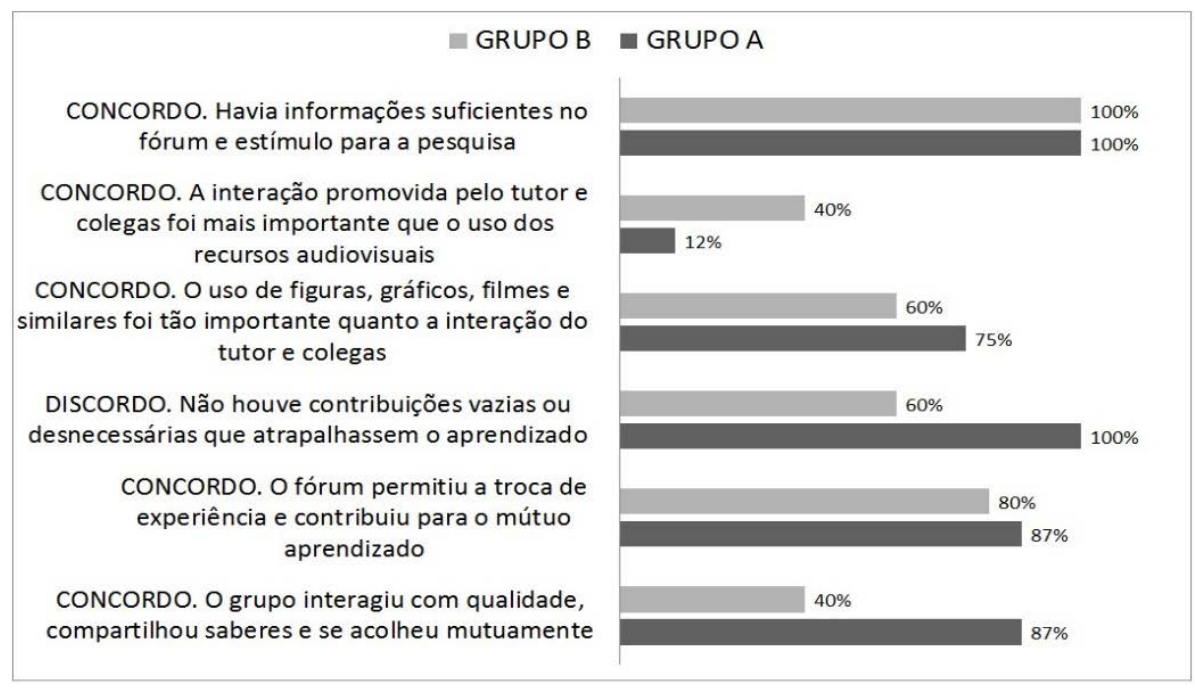

Na sequencia, apresentamos a metodologia utilizada neste levantamento da literatura. 


\section{ESTRATÉGIAS DE REVISÃo SISTEMÁticA E ANÁlISE DA LITERATURA}

Optamos pela revisão sistemática, definida por Broome (2006, apud Botelho et al., 2011) como um método investigativo específico que resume o passado da literatura empírica ou teórica para fornecer uma compreensão mais abrangente de um fenômeno particular.

A primeira ação foi definir a questão central, que precisa estar clara e bem formulada para nortear o trabalho de pesquisa (Sampaio e Mancini, 2007, p. 85). Assim, elaboramos a seguinte questão: Atividades de ensino e aprendizagem baseadas em flexibilidade cognitiva podem facilitar a construção do conhecimento em fóruns de discussão?

A seguir, elaboramos as palavras-chave e os termos relacionados utilizados na expressão de busca, que aparecem entre parênteses: $1^{\circ}$ ) atividades de aprendizagem (learning activities, atividades de ensino e aprendizagem); $2^{\circ}$ ) flexibilidade cognitiva (cognitive flexibility); $3^{\circ}$ ) fórum de discussão (discussion forum). Em seguida, escrevemos a expressão de busca, reunindo os grupos de descritores entre parênteses e delimitando pelas aspas aqueles que deviam ser localizados na ordem sintática determinada.

- (learning activities OR atividades de aprendizagem) AND ("cognitive flexibility" OR "flexibilidade cognitiva”) AND (discussion forum OR fórum de discussão).

Em alguns mecanismos de busca foi necessário adaptar a expressão. Nas bases de dados que apresentaram poucos documentos preferimos ampliar a pesquisa suprimindo descritores e refazendo a busca em ciclos, na seguinte ordem: $1^{0}$ ) fórum de discussão (discussion forum) e $2^{0}$ ) atividades de aprendizagem (learning activities, atividades de ensino e aprendizagem). Este procedimento permitiu avaliar a pertinência de documentos antes excluídos, mantendo-se um bom número de resultados disponíveis para as etapas seguintes.

Utilizamos como fonte de pesquisa 12 bases de dados, resultando em um total de 224 documentos encontrados. A escolha da base de dados seguiu três parâmetros: $\left.1^{\circ}\right)$ ao menos uma fonte de periódicos de base internacional, suficientemente vasta e sem restrições de busca e acesso, a exemplo da CAPES; $2^{\circ}$ ) um leque diversificado de bibliotecas virtuais nacionais, de reconhecido prestígio em pesquisa e divulgação científica e $3^{\circ}$ ) uma fonte de literatura não controlada por editores científicos ou comerciais, denominada no meio acadêmico como "literatura cinzenta", a exemplo do Google Acadêmico.

Na sequência, definimos os seguintes critérios de exclusão: documento repetido; com idioma diferente do inglês, português ou espanhol; ensaio clínico ou laboratorial não relacionado à flexibilidade cognitiva; sem texto completo ou pago. Após a leitura dos títulos e resumos, restringimos o levantamento da literatura para 56 
documentos, que foram lidos integralmente. As bibliotecas virtuais da UFF, UFABC, UnB e UNIFESP não retornaram resultados, mesmo simplificando-se a busca para a palavra-chave "flexibilidade cognitiva". Resumimos os resultados no quadro 1.

Dentre os documentos selecionados para leitura integral, destacamos que $40 \%$ das obras foram produzidas nos últimos quatro anos (entre 2013 e 2016) e que $48 \%$ são internacionais, provenientes de revistas de reconhecido prestígio acadêmico.

Quadro 1. Resultado obtido na consulta às bases de dados

\begin{tabular}{|c|c|c|c|c|}
\hline Base de dados & Tipo de doc & Encontrados & Excluídos & Incluídos \\
\hline \multirow{3}{*}{ UFBA (pergamum) } & Artigo & 3 & o & 3 \\
\hline & Livro & 1 & 1 & $\mathrm{O}$ \\
\hline & TOTAL & 4 & 1 & 3 \\
\hline \multirow{2}{*}{ UFG (Sibi) } & Artigo & 1 & 1 & 0 \\
\hline & TOTAL & 1 & 1 & $\mathrm{O}$ \\
\hline \multirow{4}{*}{ UFSC (pergamum) } & Artigo & 2 & 2 & $\mathrm{O}$ \\
\hline & Dissertação & 17 & 17 & 0 \\
\hline & Tese & 7 & 7 & $\mathrm{O}$ \\
\hline & TOTAL & 26 & 26 & 0 \\
\hline \multirow{5}{*}{ UFRGS (SABi) } & Artigo & 3 & 1 & 2 \\
\hline & Dissertação & 16 & 16 & 0 \\
\hline & Livro & 2 & 2 & $\mathrm{O}$ \\
\hline & \begin{tabular}{|l|} 
Monografia \\
\end{tabular} & 3 & 1 & 2 \\
\hline & TOTAL & 24 & 20 & 4 \\
\hline \multirow{4}{*}{ UNICAMP (acervus) } & E-book & 3 & 3 & 0 \\
\hline & Dissertação & 1 & O & 1 \\
\hline & Tese & 7 & 6 & 1 \\
\hline & TOTAL & 11 & 9 & 2 \\
\hline \multirow{6}{*}{ USP (dedalus) } & Artigo & 2 & 1 & 1 \\
\hline & Dissertação & 3 & o & 3 \\
\hline & Tese & 7 & o & 7 \\
\hline & Livro & 1 & 1 & $\mathrm{O}$ \\
\hline & Resumo & 6 & 6 & $\mathrm{O}$ \\
\hline & TOTAL & 19 & 8 & 11 \\
\hline \multirow{4}{*}{ Periódicos CAPES } & Artigo & 32 & 4 & 26 \\
\hline & Dissertação & 2 & 2 & 0 \\
\hline & Recurso textual & 1 & 1 & $\mathrm{O}$ \\
\hline & TOTAL & 35 & 13 & 22 \\
\hline
\end{tabular}




\begin{tabular}{|c|l|c|c|c|}
\hline \multirow{1}{*}{ Base de dados } & Tipo de doc & Encontrados & Excluídos & Incluídos \\
\hline \multirow{5}{*}{ Google Acadêmico } & Artigo & 38 & 35 & 3 \\
\cline { 2 - 5 } & Dissertação & 39 & 29 & 10 \\
\cline { 2 - 5 } & Tese & 12 & 12 & 0 \\
\cline { 2 - 5 } & Livro & 6 & 5 & 1 \\
\cline { 2 - 5 } & Resumo & 2 & 2 & 0 \\
\cline { 2 - 5 } & Monografia & 7 & 7 & 0 \\
\cline { 2 - 5 } & TOTAL & 104 & 90 & 14 \\
\hline \multirow{2}{*}{ TOTAL GERAL DE DOCUMENTOS } & 224 & 168 & 56 \\
\hline
\end{tabular}

\section{ANÁLISE E INTERPRETAÇÃO DOS RESULTADOS}

Dos 56 documentos selecionados para leitura integral, 32 foram considerados pertinentes para o desenvolvimento das reflexões apontadas neste estudo e 24, não pertinentes. Os motivos são analisados a seguir.

\section{Documentos selecionados como não pertinentes}

As obras dos pesquisadores Abujadi (2013), Balestra (2012), Cavallini et al. (2015), Colzato et al. (2009), Cunha et al. (2010), D’alcante (2010), Farmer e Eakman (1995), Gligorovic e Buha (2013), Nascimento (2015), Ohira et al. (2011), Pinto (2011), Samuelson et al. (2012), Sylwan (2001), Van Uem et al. (2016), Wolf et al. (2016) e Zimmermann (2014) foram excluídas desta investigação literária por centrarem-se em pelo menos um dos seguintes aspectos: tratarem de análise clínico-laboratorial, estudo sistemático de interação medicamentosa ou observação exploratória experimental dos efeitos nas funções cerebrais em circunstâncias clínicas. A abordagem da flexibilidade cognitiva, nestes estudos neurocientíficos das FE, tiveram uma especificidade diferente da objetivada neste trabalho.

Silva et al. (2011), Salles (2009) e Rosatelli (1999) realizaram uma abordagem geral das teorias da aprendizagem, para abordar os AVEA e sustentar o estudo específico de seus casos. A TFC foi apenas citada pontualmente em uma página, sem mais detalhamentos ou referência à ferramenta fórum de discussão.

Os estudos de Dalledone (2003), Schubert e Andersson (2013), Klopfer et al. (2005) e Neyrinck et al. (2006) também foram excluídos, após leitura integral, pois os pesquisadores abordaram seus objetos específicos de estudo acerca do desempenho cognitivo e da flexibilidade do pensamento sem estabelecer uma relação clara com a TFC. De forma similar, Leite (2010) apenas cita superficialmente a TFC como uma teoria de aprendizagem que pode ser aplicada às TIC e à Web 2.o. 


\section{Documentos selecionados como pertinentes}

As pesquisas de Bernardes (2015), Debiasi (2013), Dias (2014), Zuk et al. (2014), Paleari (2013), Portowitz et al. (2014), Messina (2015), Toledo (2006) e Zuanetti (2015) apontam que a Flexibilidade Cognitiva é um componente das FE do Lobo Frontal e permite ao sujeito adaptar cognitivamente suas perspectivas, formas de pensamento ou conceitos preestabelecidos em face de mudanças externas, novas exigências ou transformações. A neuroimagem funcional e a estimulação magnética transcraniana são exemplificadas como meios de se capturar a flexibilidade de pensamento ocorrendo no CPF, além de outras áreas do lobo frontal, no momento de execução de tarefas, o que evidencia o desenvolvimento cognitivo em atividades baseadas no estímulo das funções executivas. Neste levantamento também verificamos que certos problemas neuropsicológicos do comportamento, a exemplo dos transtornos obsessivos, depressivos, compulsivos, de atenção e hiperatividade, podem afetar a capacidade de alternar o estado mental e flexibilizar o pensamento.

Os pesquisadores Aleixo et al. (2008), Balcytiene (1999), Brito et al. (2006; 2007), Carvalho (2002; 2008), Chieu (2007), Goudouris et al. (2013), Lozada (2013), Newswander e Newswander (2012), Pessoa e Nogueira (2009), Pires e Leão (2009), Strobel et al. (2008), Tonietto et al. (2007) e Wu e Puntambekar (2012) apresentam a TFC como uma teoria construtivista de ensino e aprendizagem desenvolvida por Spiro e colaboradores na década de 8o, voltada para o nível avançado do conhecimento e direcionadas às situações complexas e pouco estruturadas, já abordadas anteriormente. Para Chieu (2007), a TFC faclita a apropriação de conceitos não familiares a partir dos familiares, por meio de analogia e transferência de domínios do conhecimento. Aponta, ainda, um importante princípio da TFC: a simplificação ou estruturação exagerada da apresentação de problemas pode levar o estudante a cometer erros conceituais de generalização e enviesamento quando tenta aplicar o mesmo esquema resolutivo aos domínios complexos e mal estruturados. Balcytiene (1999) pondera que a quantidade e variedade de hipertextos educacionais disponíveis na rede com a capacidade de permitir os múltiplos atravessamentos conceituais ainda é incompatível com a sua importância.

Araujo (2009) apresenta modelos pedagógicos e estratégias de ensino e aprendizagem em AVEA que situam o estudante como sujeito ativo na experiência educacional, abordando como a educação a distância pode tornar-se um ambiente profícuo de planejamento e uso sociocultural de ferramentas da tecnologia educacional para propiciar a gestão do conhecimento, bem como aperfeiçoar o potencial de aprendizagem, a interação e a produção, do ponto de vista do desenho instrucional. Aponta a Flexibilidade Cognitiva como uma teoria da aprendizagem construtivista vinculada aos hipertextos, promotora de perspectivas múltiplas, não lineares, transversais, complexas e interligadas, bem como o termo "atividades autênticas", atribuída a Dabbagh e Bannan-Ritland (2005, apud Araujo, 2009), como tarefas ou atividades de aprendizagem que encorajam os estudantes a explorarem 
diferentes estratégias de estudo de casos e resolução de problemas; colaborar, refletir, negociar, comparar, investigar e gerar hipóteses.

A Aprendizagem Baseada em Problemas (ABP) é apresenta por Fontes (2013) como um método guiado de educação ativa na qual situações-problema não triviais ou com solução única são apresentadas aos estudantes para resolução colaborativa, dedicando um capítulo às teorias que abordam este tipo de aprendizagem ativa, centrada no estudante, social-construtivista-interacionista. Traça um relacionamento íntimo com a TFC ao afirmar que os casos devem ser complexos e mal estruturados. Reforça ainda que, apesar de coletiva, atividades desta natureza permitem uma avaliação individual, uma vez que os sujeitos assumem parcelas de contribuição no desenvolvimento da tarefa.

Van de Ven et al. (2015) abordam o uso de jogos de computadores, bem como exercícios de flexibilidade cognitiva de reabilitação relacionados à execução de tarefas cotidianas, por um grupo de pacientes idosos (até 80 anos) que sofreram AVC e passaram por reabilitação, possuindo prejuízos cognitivos evidenciados por avaliação médica especifica (excluindo os portadores de dependências químicas e outras doenças cognitivas graves, a exemplo das neurodegenerativas ou psiquiátricas). Os pesquisadores, em sessões de treinamento dos três domínios cognitivos das FE (atenção, raciocínio e memória de trabalho), observaram melhorias no intuito de remediar os prejuízos da falta de flexibilidade cognitiva.

Dong et al. (2014) aponta que o uso inapropriado das tecnologias digitais também pode resultar em perdas no desenvolvimento cognitivo, referindo-se ao uso vicioso da internet como um transtorno cognitivo e comportamental, no qual os sujeitos apresentam prejuízos na flexibilidade cognitiva devido ao uso excessivo ou descontrolado, apresentando consequências negativas nos aspectos psicológico, social e laboral, embora, segundo a autora, este não esteja incluído no Manual de Diagnósticos e Estatística de Transtornos Psicológicos. A Flexibilidade Cognitiva é definida pela autora como um processo mental que exige do sujeito a capacidade de se adaptar, rápida e eficientemente, a situações às quais seja necessária a reconfiguração mental ou a alternância sequencial, para completar com êxito uma determinada tarefa.

Lima (2002), na primeira metade da dissertação, preocupou-se em analisar comparativamente as funcionalidades de diversos tipos de AVEA, tais como o WebAula, o TelEduc, a UVB.BR, o AulaNet e o Alumini Gestum. Na sequência, aborda mais especificamente as teorias de aprendizagem focadas no desenho de um framework, definido por ela como um "modelo conceitual que descreve um sistema ou processo, frequentemente em termos abstratos, para que o mesmo possa ser compreendido em profundidade, permitindo a avaliação de suas características e funcionalidades" e composto de três itens: Princípio, Autoria do Curso e Realização do Curso. A autora classifica os ambientes de suporte à aprendizagem cooperativa da seguinte forma: 1) teorias de aprendizagem fundamentadoras, 2) modelos de cooperação ou tipos de tarefas para aprendizagem cooperativa adequado ao 
ambiente, independente do domínio de assunto ensinado, 3) domínios, 4) tipo e grau de interação possibilitado pelo ambiente, 5 ) atividades de trabalho cooperativo, 6) plataformas, 7) designação de papéis exercidos pelos usuários e 8) relação com outras áreas de pesquisa.

Pacheco (2013) estrutura o seu trabalho em duas partes. A primeira é constituída pelo enquadramento teórico, na qual discute o conhecimento mediado pela tecnologia e a influência da globalização como um fenômeno de profunda transformação sociocultural, criadora das chamadas sociedades da informação ou do conhecimento, na qual "o saber se tornou o capital econômico mais competitivo e as tecnologias invadiram todos os setores da vida humana". Ainda neste capítulo é abordada a necessidade de prover ferramentas pedagógicas de estímulo à educação ao longo da vida (lifelong learning), a flexibilização do papel de professor e estudante, bem como as teorias da aprendizagem, dentre elas a flexibilidade cognitiva. O pesquisador conclui com uma análise dos AVEAS e do papel dos vários atores estruturantes dos cursos a distância, especialmente o Tutor. A segunda parte destinou-se à apresentação do estudo empírico de um curso online de mestrado de uma universidade privada portuguesa. Junior (2010) também aborda as principais teorias da aprendizagem voltadas para a educação tecnológica, incluindo a TFC.

\section{CONSIDERAÇÕES FINAIS}

Em fóruns de discussão, nos cursos de educação a distância, normalmente os estudantes são estimulados por tutores e professores a construir o conhecimento de determinados assuntos por meio da conjugação comum de saberes e pontos de vista. Nesta tarefa, a qualidade da interação e a representação do pensamento são fundamentais.

A partir dos estudos empíricos de Carvalho sobre a aplicação da TFC nos AVEA (2000; 2002; 2008), retomamos a nossa questão de pesquisa sugerindo que a elaboração de atividades para fóruns de discussão baseada na forma flexibilizada de apresentação da informação possui relevância cognitiva. No entanto, a revisão da literatura identificou a escassez de estudos dedicados a explorar a discussão de casos ou situações-problema no contexto da vida real em fóruns de discussão, conforme abordado conceitualmente pela TFC, o que torna a questão central inconclusiva, apesar dos resultados positivos de nosso estudo empírico. Um dos motivos potenciais pode estar relacionado ao fato de muitos educadores não terem se habilitado para processar a desconstrução e reconstrução conceitual de seus objetos de ensino para múltiplas representações da realidade, uma vez que somos acostumados a gerar respostas e não pressupostos; a apresentar soluções e não pontos de vista. Ainda sentimos grande desconforto em praticar o ensino baseado em perguntas e autodescobertas, preferindo as respostas diretas e os exercícios de reflexão linear.

De fato, a aprendizagem se faz por meio de uma complexa relação de funções cognitivas superiores que parece envolver uma fina percepção sobre o pensamento e 
as relações sociais no desenvolvimento das habilidades motora, afetiva e psicológica. Assim, consideramos que a aprendizagem seja uma etapa intrapessoal baseada na tomada de consciência crítica de si, do mundo, dos sujeitos e das interferências possíveis, abrangendo um conjunto de habilidades cognitivas de alta ordem, responsável pela coordenação de respostas adaptativas diante de situações novas e/ ou complexas.

A Flexibilidade Cognitiva foi uma característica centralmente apontada tanto na TFC quanto nas FE, no sentido de promover o desenvolvimento da capacidade de flexibilizar o pensamento e os construtos cognitivos que promovem o conhecimento dos estudantes, ajudando-os a compreender melhor as convenções sociais e interferências tecnológicas que ligam textos verbais e não verbais às mais variadas expressões matemáticas, gráficas, diagramáticas e esquemáticas (Pessoa e Nogueira, 2009; Spiro et al., 1992). Assim, a apreensão dos caminhos resolutivos de um caso ou situação-problema é tão significativa ao aprendizado quanto à resolução do problema em si.

Vigotski (2008) observa que a simples presença de um problema não é suficiente para provocar o processo de assimilação de um conceito. Ausubel (2003) também aponta que o ensino por descoberta, sem a devida mediação, transforma o aluno em um pedagogo de si próprio, não resultando em aprendizagem significativa. Entretanto, ambos concordam que o aprendizado deve apresentar desafios, tarefas e exigências novas ao estudante para estimular o intelecto.

Liu et al. (2016) e Carbonella et al. (2016) apontam que pessoas com inflexibilidade cognitiva, provocada por traumas cerebrais ou disfunções neuropsicológicas, por exemplo, apresentam dificuldades para inibir um conjunto de respostas prévias, comutar tarefas e adaptar novos conhecimentos aos já existentes, sofrendo confusão mental. Liu et al. (2016) explicam que, em testes neuropsicológicos, este comportamento não foi observado em indivíduos que apresentam alta flexibilidade cognitiva. Desta forma, acreditamos que a elaboração de atividades flexibilizadas para fóruns de discussão é muito importante para estimular o desenvolvimento desta capacidade mental, promovendo a reflexão e a representação do pensamento. Neste sentido, a TFC pode colaborar com os AVEA fornecendo a base teórica para a abordagem diversificada das fontes de informação, por meio de música, infográficos, poemas, imagens, dentre outros recursos verbais e não verbais, bem como a resolução de casos ou situações-problema capazes de estimular a argumentação, o debate e a socioconstrução do conhecimento.

Como observado por Carvalho (2000; 2002; 2008) e Junior (2010), principalmente, a TFC de Spiro e colegas possui algumas limitações que devem ser respeitadas: A) sua aplicação se faz em um nível específico e intermediário de aprendizagem, a avançada, que necessita de um conjunto de conhecimentos prévios para suportar os novos; B) exige do professor, bem como da equipe instrucional, a capacidade de desconstruir os objetos de aprendizagem e reconstruir atividades com múltiplas representações do conhecimento; C) facilitar a discussão de casos 
ou situações-problema como meio principal de desenvolvimento e avaliação do conhecimento; D) por ser uma teoria adequada ao trabalho em contextos complexos e pouco estruturados, domínios do conhecimento concretos e operacionais não são bons candidatos ao trabalho com a TFC. Assim, os professores devem resistir às respostas diretas, ao ensino expositivo e à apresentação simplificada do conhecimento.

Visando contribuir com os estudos futuros sobre os mecanismos cognitivos do comportamento humano e a produção colaborativa do conhecimento, sugerimos o aprofundamento teórico-comparativo das obras de Vigotski, Ausubel e Spiro, bem como a investigação mais aprofundada dos aspectos neuropsicológicos cognitivos da aprendizagem. Propomos, também, a necessidade de se construir modelos de codificação e medição da qualidade de interação entre os estudantes que participam de fóruns de discussão, aferindo-se a efetividade da produção de conhecimentos baseados na socialização dos pontos de vista e na mediação do saber.

\section{REFERÊNCIAS BIBLIOGRÁFICAS}

Abujadi, C. (2013). Estimulação Magnética Transcraniana em indivíduos com autismo. (Dissertação mestrado), São Paulo: FMUSP.

Aleixo, A. A. et al. (2008). FlexQuest: potencializando a WebQuest no Ensino de Química, Faced, 14, 119-133.

Araujo, E. M. (2009). Design instrucional de uma disciplina de pós-graduação em Engenharia de Produção: uma proposta baseada em estratégias de aprendizagem colaborativa em ambiente virtual. (Dissertação Mestrado). São Carlos: EESC/USP.

Ausubel, D. P. (2003). Aquisição e Retenção de Conhecimentos: Uma Perspectiva Cognitiva. Ligia Teopisto (trad.), ( $\left.1^{\mathrm{a}} \mathrm{ed}.\right)$, Lisboa: Plátano.

Balcytiene, A. (1999). Exploring individual processes of knowledge construction with hypertext. Instructional Science, 303328.

Balestra, A. G. (2012). Caracterização de funções neurocognitivas em pacientes com Transtorno Depressivo Maior com e sem sintomas psicóticos. (Dissertação Mestrado), Ribeirão Preto: FMRP/USP.
Bernardes, E. T. (2015). Estudo das relações entre maus tratos na infância, prejuízo em funções executivas e transtornos do comportamento disruptivo em uma amostra comunitária de crianças. (Dissertação Mestrado). São Paulo: FMUSP.

Botelho, L. L. R., Cunha, C. A. C., e Macedo, M. (2011). O Método da revisão integrativa nos estudos organizacionais. Gestão $e$ sociedade, Belo Horizonte: UFMG, 5(11), 121-136.

Brito, R. F. et al. (2006). Desenvolvendo Objetos de Aprendizagem SCORM aplicando a Arquitetura da Informação e Teoria da Flexibilidade Cognitiva. Informática na Educação: Teoria \& prática, 9(1), 97-108.

- (2007). Desenvolvimento de cenários digitais interoperáveis para aprendizagem baseada em problemas. (Dissertação mestrado). Florianópolis: UFSC

Carvalho, A. A. A. (2008). Os LMS no Apoio ao Ensino Presencial: dos conteúdos às interacções. Revista Portuguesa de Pedagogia, 101-122. 
. (2002). Promover a Flexibilidade Cognitiva em Níveis Avançados do Conhecimento. Faced, 6, 25-46.

(2000). A representação do conhecimento segundo a Teoria da Flexibilidade Cognitiva. Revista Portuguesa de Educacão, 169-184. Recuperado de http://repositorium. sdum.uminho.pt/bitstream/1822/488/1/ AnaAmeliaCarvalho.pdf

Carbonella, J. et al. (2016). Examining the Link Between Hoarding Symptoms and Cognitive Flexibility Deficits. Elsevier, Behavior therapy, 47(2), 262 -273;

Cavallini, E. et al. (2015). Self-help memory training for healthy older adults in a residential care center: specific and transfer effects on performance and beliefs. International Journal of Geriatric Psychiatry, 870-880.

Chieu, V. M. (2007). An Operational Approach for Building Learning Environments Supporting Cognitive Flexibility. Educational Technology \& Society, 32-46.

Colzato, L. S. et al. (2009). Recreational cocaine polydrug use impairs cognitive flexibility but not working memory. Psychopharmacology, p. 225-234.

Cunha, P. J. et al. (2010). The frontal assessment battery (FAB) reveals neurocognitive dysfunction in substancedependent individuals in distinct executive domains: Abstract reasoning, motor programming, and cognitive flexibility. Elsevier, Addictive Behaviors, 35, 875-881.

D’Alcante, C. C. (2010). Características neuropsicológicas no transtorno obsessivo compulsivo e seu impacto na resposta ao tratamento. (Dissertação Mestrado), São Paulo: FMUSP.

Dalledone, S. B. S. (2003). Ambiente de apoio ao processo ensino-aprendizagem na internet para desenvolvimento de projetos educacionais. (Dissertação mestrado). Florianópolis: UFSC.
Debiasi, N. S. F. (2013). Um estudo de associação entre sintomas de déficit de atenção/hiperatividade e medidas neuropsicológicas de flexibilidade cognitiva e atenção seletiva em crianças. (Monografia especialização). Porto Alegre: UFRGS.

Diamond, A. et al. (2007). Preschool Program Improves Cognitive Control. Science, 318, 1387-1388;

Dias, C. M. (2014). Avaliação Neuropsicológica das Funções Executivas em um Adulto com Sintomas de Ansiedade. (Monografia especialização). Porto Alegre: UFRGS.

Dong, G. et al. (2014). Cognitive flexibility in internet addicts: fMRI evidence from difficult-to-easy and easy-to-difficult switching situations. Addictive Behaviors, 677-683.

Farmer, J. E., e Eakman, A. M. (1995). The relationship between neuropsychological functioning and instrumental activities of daily living following acquired brain injury. Applied Neuropsychology, 107115.

Fontes, L. M. O. (2013). Uma Arquitetura Multiagente de Apoio à Aprendizagem Baseada em Problema. (Dissertação Mestrado). Mossoró: UFERSA/UERN.

Gligorovic, M., e Buha, N. (2013). Conceptual abilities of children with mild intellectual disability: Analysis of Wisconsin Card Sorting Test performance. Journal of Intellectual \& Developmental Disability, 134-140.

Goudouris, E. S. et al. (2013). Tecnologias de Informação e Comunicação e Ensino Semipresencial na Educação Médica. Revista Brasileira de Educação Médica, Rio de Janeiro, 396-407.

Junior, C. A. M., e Melo, L. B. R. (2011). Integração de Três Conceitos: Função Executiva, Memória de Trabalho e Aprendizagem. Psicologia: Teoria $e$ Pesquisa, 27(3), 309-314. 
Junior, J. B. B. (2010). As abordagens educacionais: das perspectivas comportamentalistas à aprendizagem em rede. Concepção, Avaliação e Dinamização de um Portal Educacional de WebQuests em Língua Portuguesa. (Tese Doutorado). Braga: Universidade do Minho, cap. 2, pp. 41-97.

Klopfer, E. et al. (2005). Using Palm Technology in Participatory Simulations of Complex Systems: A New Take on Ubiquitous and Accessible Mobile Computing. Journal of Science Education and Technology, 14(3), 285-297.

Leite, B. S. (2011). Uso das tecnologias para o ensino das ciências: a web 2.0 como ferramenta de aprendizagem. (Dissertação Mestrado). Recife: UFRP.

Leite, B. S., e Leão, M. B. C. (2010). Contribuição da web 2.0 no ensino de química. Congreso Iberoamericano de Informática Educativa, 1, (pp. 99-106). Santiago de Chile.

Lima, L. S. (2002). Proposta de um Framework de apoio ao desenvolvimento de cursos a distância baseados na abordagem sócio-histórica de Vigotski. Dissertação (Mestrado). Florianópolis: UFSC;

Liu, H. et al. (2016). The effect of cognitive flexibility on task switching and language switching. The International Journal of Bilingualism, 2O(5), 563-579.

Lozada, C. O. (2013). Direito Ambiental: relações jurídicas modeladas pela Matemática visando uma formação profissional crítica e cidadã dos bacharelandos em Engenharia Ambiental. (Tese Doutorado). São Paulo: FEUSP.

Messina, B. (2015). Impulso sexual excessivo, aspectos neuropsicológicos no estado de vigília e pós-estímulo sexual: estudo experimental. (Dissertação Mestrado). São Paulo: FMUSP.

Moreira, L. R. (2014). Manual de revisão bibliográfica sistemática integrativa: a pesquisa baseada em evidências. Belo Horizonte: Anima Educação.

Morton, J. B. et al. (2013). Funções executivas. Enciclopédia sobre o Desenvolvimento na Primeira Infância. Ontário: CEECD.

Nascimento, M. A. (2015). Estratégias de Remediação Cognitiva no Envelhecimento. (Dissertação mestrado). Rio de janeiro: UFRJ.

Newswander, L. K., e Newswander, C. B. (2012). Encouraging Cognitive Flexibility and Interdisciplinarity in Public Administration Programs. Administration \& Society, 285-309.

Neyrinck, B. et al. (2006). Cognitive, Affective and Behavioral Correlates of Internalization of Regulations for Religious Activities. Motivation and Emotion, 323-334.

Ohira, H. et al. (2011). Chronic stress modulates neural and cardiovascular responses during reversal learning. Neuroscience, 193-204.

Pacheco, R. M. V. N. (2013). Ensinar $e$ aprender no ensino superior através do ensino a distância: o perfil pedagógico do professor/tutor e o perfil de aprendizagem dos estudantes em cursos b-learning. (Dissertação Mestrado). Algarve: Universidade do Algarve.

Paleari, A. P. G. (2013). Crianças com queixa atencional e alterações psicomotoras: estudo comparativo. (Dissertação Mestrado). São Paulo: FOBUSP.

Pessoa, T., e Nogueira, F. (2009). Flexibilidade Cognitiva nas vivências e práticas educativas: Casebook para a formação de professores. In A. D. Nascimento e T. M. Hetkowski (Orgs.), Educação e contemporaneidade: pesquisas científicas e tecnológicas [online]. Salvador: EDUFBA.

Pinto, L. K. (2011). Um estudo com o Psicodiagnóstico de Rorschach sobre o funcionamento psíquico de pacientes que realizaram tratamento de obesidade. 
(Dissertação mestrado), São Paulo: IPUSP.

Pires, C. A., e Leão, M. B. C. (2009). Ambiente Virtual de Estudo "Escola Virtus" e o Podcasting Multimídia como recursos no processo de ensino-aprendizagem. Revista Latinoamericana de Tecnología Educativa (RELATEC), 8(2), 3957.

Portowitz, A. et al. (2014). In Harmony: A technology-based music education model to enhance musical understanding and general learning skills. International Journal of Music Education, 32(2), 242260.

Rosatelli, M. C. (1999). Um Ambiente Inteligente para Aprendizado Colaborativo no Ensino a Distância Utilizando o Método de Casos. (Tese Doutorado). Florianópolis: UFSC.

Salles, B. W. (2009). Desenvolvimento de uma base de conhecimento de casos clínicos de pacientes portadores de desordem temporomandibular, como forma de organização do conhecimento e auxilio no diagnóstico. Tese (Doutorado). Florianópolis: UFSC.

Sampaio, R. F., e Mancini, M. C. (2007). Estudos de revisão sistemática: um guia para a síntese criteriosa da evidência científica. Revista Brasileira de Fisioterapia, 11(1), 83-89.

Samuelson, K. W. et al. (2012). Relationships Between Maternal Emotion Regulation, Parenting, and Children's Executive Functioning in Families Exposed to Intimate Partner Violence. Journal of Interpersonal Violence, 3532-3550.

Schubert, T., e Andersson, M. (2013). Old is Gold? The Effects of Employee Age on Innovation and the Moderating Effects of Employment Turnover. Centre for Innovation, Research and Competence in the Learning Economy (CIRCLE), Lund: Universidade de Lund, 1-27.

Silva, M. P. et al. (2011). Estratégias de ensino para o planejamento didático pedagógico de um sistema de ensino a distância mediado pelo ambiente virtual de ensino aprendizagem. Em A. Y. Youssef e A. Ramirez, A., Social Software in Higher Education: Pedagogical Models and Universities Strategies Palhoça: Editora Unisul.

Spiro, R. J. et al. (1987). Knowledge Acquisition for Application: Cognitive Flexibility and Transfer in Complex Content Domains. Technical Report No. 409. Center for the Study of Reading, National Institute of Education (U.S.), U.S. Army Research Institute for the Behavioral and Social Sciences. University of Illinois at Urbana-Champaign. Recuperado de http://files.eric.ed.gov fulltext/ED287155.pdf (1992). Cognitive Flexibility, Construtivism and Hypertext: Random Access Instruction for Advanced Knowledge Acquisition in Ill-Structured Domains. In T. M. Duffy e D. H. Jonassen, Constructivism and the Techonology of Instruction: A Conversation, (57-75). Hillsdale, NJ: Lawrence Erilbaum,

Strobel, J. et al. (2008). The evolution of a collaborative authoring system for nonlinear hypertext: A design-based research study. Computers \& Education, 67-85.

Sylwan, R. P. (2001). Algumas contribuições experimentais ao problema da habilidade inibitória em tarefas com interrupção sinalizada. (Tese Doutoramento), Ribeirão Preto: FFCLRP/USP.

Toledo, M. M. (2006). Comparação do diagnóstico e resposta a um treino de atenção em crianças com os subtipos de transtorno e déficit de atenção/ hiperatividade. (Tese Doutorado). Campinas: Unicamp.

Tonietto, L. et al. (2007). Aquisição Inicial do Léxico Verbal e Aproximações Semânticas em Português. Psicologia: Reflexão $e$ Crítica, 114-123.

Van de Ven, R. M. et al. (2015). The effect of computer-based cognitive flexibility training on recovery of executive function 
after stroke: rationale, design and methods of the TAPASS study. BMC Neurology, Amisterdã: Universidade de Amisterdã, 1-12.

Van Uem, J. M. T. et al. (2016). Quantitative Timed-Up-and-Go Parameters in Relation to Cognitive Parameters and HealthRelated Quality of Life in Mildto-Moderate Parkinson's Disease. PLOS ONE, 1-15.

Vygotsky, L. S. (1996). A formação social da mente. Rio de Janeiro: Martins Fontes; . (2008). Pensamento e linguagem.

Jefferson Luiz Camargo (trad.), ( $4^{\mathrm{a}}$ ed.), São Paulo: Martins Fontes.

Wolf, T. J. et al. (2016). Combined CognitiveStrategy and Task-Specific Training Affects Cognition and Upper-Extremity Function in Subacute Stroke: An Exploratory Randomized Controlled Trial.
The American Journal of Occupational Therapy, 70 (2), 1-10.

Wu, H., e Puntambekar, S. (2012). Pedagogical Affordances of Multiple External Representations in Scientific Processes. J. Sci Educ Technol, 754-767.

Zimmermann, N. (2014). Working memory training and poetry-based stimulation programs: Are there differences in cognitive outcome in healthy older adults? NeuroRehabilitation, 159-170.

Zuanetti, P. A. (2015). Consequências da obesidade infantil nas habilidades cognitivas envolvidas na aprendizagem escrita. (Tese Doutorado). Ribeirão Preto: FFLRPUSP.

Zuk, J. et al. (2014). Behavioral and Neural Correlates of Executive Functioning in Musicians and Non-Musicians. PLoS ONE, 9(6), 1-14.

\section{PERFIL ACADÊMICO E PROFISSIONAL DOS AUTORES}

Eduardo Rodrigues da Silva. Mestrando em Ciências Humanas e Sociais na linha de pesquisa sobre Cultura, Comunicação e Dinâmica Social, pela Universidade Federal do ABC (UFABC). Especialista em Educação a Distância pela Universidade Federal Fluminense (UFF). Graduado e Licenciado em Letras-Linguística pela Universidade de São Paulo (USP). Educador voluntário na Universidade Federal de São Paulo (UNIFESP). Tutor em cursos de educação a distância.

E-mail: silva.eduardo@ufabc.edu.br

Silvia Dotta. Doutora em Educação pela Faculdade de Educação da USP. É docente da Universidade Federal do ABC e coordenadora de Audiovisual do Núcleo de Tecnologia Educacional. É membro do grupo de pesquisa INTERA onde realiza pesquisas com ênfase em Educação a Distância, Tecnologias Educacionais e Popularizacão das Ciências Antárticas.

E-mail: silvia.dotta@ufabc.edu.br

\section{ENDEREÇO DOS AUTORES}

Universidade Federal do ABC (UFABC)

Av. dos Estados, 5001

Santa Terezinha, Santo André, SP (Brasil)

09210-580 
Data de recebimento do artigo: 30/10/2016

Data de aceite do artigo: 26/12/2016

\section{Como citar este artigo:}

Da Silva, E. R., e Dotta, S. (2018). Interfaces da flexibilidade cognitiva e da aprendizagem em fóruns de discussão. RIED. Revista Iberoamericana de Educación a Distancia, 21(1), pp. 303-322. doi: http://dx.doi.org/10.5944/ried.21.1.17496 